\title{
ANÁLISE DE PREVALÊNCIA DE RETINOPATIA DIABÉTICA EM PACIENTES ATENDIDOS NO HOSPITAL DE OLHOS DO TOCANTINS NO ANO DE 2020
}

\author{
ANALYSIS OF PREVALENCE OF RETINOPATHY DIABETIC IN \\ PATIENTS TREATED INHOSPITAL TOCANTINS EYES OF THE YEAR \\ 2020
}

\author{
Eduardo Cunha Costa ${ }^{1}$ \\ Walter Helene Nunes Cazarotto ${ }^{2}$ \\ João Carlos Diniz Arraes ${ }^{3}$ \\ Rejanne Lima Arruda 4
}

\begin{abstract}
RESUMO: OBJETIVO: Analisar a prevalência de retinopatia diabética nos pacientes atendidos no Hospital de Olhos do Tocantins, localizado na cidade de Araguaína, no ano de 2020. MÉTODOS: Estudo epidemiológico realizado por meio da análise de prontuários de pacientes atendidos no Hospital de Olhos do Tocantins, da cidade de Araguaína, no ano de 2020. As variáveis analisadas foram: idade, sexo, uso de insulina, tempo de tratamento da diabetes, tipo de retinopatia diabética, hipertensão arterial e presença de catarata. Os dados foram coletados mediante acesso ao Sistema de Gestão Hospitalar Gemmius ${ }^{\circledR}$ implantado e utilizado no hospital em estudo, e em seguida realizou-se a tabulação dos dados através do aplicativo Microsoft Excel. RESULTADOS: Foram selecionados 236 prontuários de pacientes atendidos no ano 2020 com este diagnóstico. Após a análise observou-se que $48,73 \%$ dos pacientes tinham idade entre 41 a 60 anos, $47,46 \%$ tinham mais que 60 anos e $3,81 \%$ de 0 a 40 anos. Em relação ao sexo $55,08 \%$ eram indivíduos do sexo masculino e $44,92 \%$ do sexo feminino. Quanto ao uso de insulina, 41,95\% faziam uso e $58,05 \%$ não usavam. Dentre os pacientes avaliados, $65,25 \%$ apresentavam diagnóstico de hipertensão arterial e $68,22 \%$ diagnóstico de catarata. Em se tratando do tempo de tratamento de diabetes $48,56 \%$ faziam tratamento entre 6 e 15 anos, já
\end{abstract}

\footnotetext{
${ }^{1}$ Aluno de graduação do $7^{\circ}$ período curso de Medicina do Centro Universitário Presidente Antônio Carlos, da cidade de Araguaína-TO.

2 Aluno de graduação do $7^{\circ}$ período curso de Medicina do Centro Universitário Presidente Antônio Carlos, da cidade de Araguaína-TO.

3 Professor da Universidade Federal do Tocantins. Coordenador da Residência Médica em Oftalmologia do HO/UFT/HDT. Doutorado pela UFG. Diretor e Médico-oftalmologista do Hospital de Olhos do Tocantins.

${ }^{4}$ Professora Doutora do Centro Universitário Presidente Antônio Carlos, campus Araguaína- TO.
} 
em relação ao tipo de retinopatia diabética, a proliferativa mostrou-se mais relevante sendo $39,83 \%$ delas. CONCLUSÃO: Dos pacientes que faziam uso de insulina, $48,98 \%$ apresentavam retinopatia diabética proliferativa, e $43,83 \%$ tratavam a mais de 15 anos de diabetes mellitus.

Palavras chave: Estudo oftalmológico. Diabetes mellitus. Saúde pública.

ABSTRACT: OBJECTIVE: To analyze the prevalence of diabetic retinopathy in patients treated at the Hospital de Olhos do Tocantins, located in the city of Araguaína, in 2020. METHODS: Epidemiological study was conducted through the analysis of medical records ofpatients treated at the Hospital de Olhos do Tocantins, in the city of Araguaína, in 2020. The variables analyzed were: age, gender, insulin use, time of treatment of diabetes, type of diabetic retinopathy, hypertension and presence of cataracts. Data were collected through access to the Gemmius Hospital Management System ${ }^{\circledR}$ implanted and used in the hospital under study, and then tabulation of the data was performed through the Microsoft Excel application. RESULTS: We selected 236 medical records of patients treated in 2020 with this diagnosis. After the analysis, it was observed that $48.73 \%$ of the patients were aged between 41 and 60 years, $47.46 \%$ were older than 60 years and $3.81 \%$ were between 0 and 40 years old. Regarding gender, $55.08 \%$ were males and $44.92 \%$ were females. Regarding the use of insulin, $41.95 \%$ did not use it and $58.05 \%$ did not use it. Among the patients evaluated, $65.25 \%$ had a diagnosis of hypertension and $68.22 \%$ were diagnosed with cataracts. Regarding the time of treatment of diabetes $48.56 \%$ were treated between 6 and 15 years, already in relation to the type of diabeticretinopathy, proliferative was more relevant, $39.83 \%$ of them. CONCLUSION: Of the patients who used insulin, $48.98 \%$ had proliferative diabetic retinopathy, and $43.93 \%$ treated more than 15 years of diabetes mellitus.

Keywords: Ophthalmologic study. Diabetes mellitus. Public health. 


\section{INTRODUÇÃO}

O diabetes mellitus (DM) é caracterizado por uma deficiência na produção de insulina ou na incapacidade da mesma de realizar as suas funções fisiológicas, acompanhado por hiperglicemia persistente. A Organização Mundial da Saúde (OMS) afirma que a hiperglicemia é o terceiro fator da causa de mortalidade prematura ficando atrás do uso de tabaco e da pressãoarterial elevada (MCLELLAN et al., 2007).

Segundo o Departamento de Enfermagem da Sociedade Brasileira de Diabetes, as complicações podem ser divididas em agudas e crônicas. Portanto, estão incluído nas manifestações clinicas agudas a hipoglicemia, cetoacidose diabética e o estado hiperosmolar hiperglicêmico e nas complicações crônicas a nefropatia, a retinopatia, doenças micro emacrovasculares.

A retinopatia é uma das diversas consequências causadas pelo diabetes e é caracterizado por microaneurismas desses capilares da retina devido a morte dos pericitos que circulam e sustentam a parede esses capilares. A sociedade Brasileira de Diabetes, em sua publicação do ano de 2015 afirma que a principal causa da diminuição da acuidade visual é o edema macular, podendo manifestar-se desde as fases iniciais da doença até em casos de pacientes com mais de vinte anos com diabetes (SBD, 2015).

De acordo com o estudo de Bosco et al (2005) a retinopatia se manifesta tanto em pacientes com diabetes tipo 1 quanto no tipo 2 e está fortemente associado ao mau controle glicêmico. A fisiopatologia das consequências micro e macro vasculares, principalmente do tecido retiniano, está associado a hiperglicemia crônica, que gera alterações sistêmicas como a diminuição do tônus muscular, aumento da permeabilidade vascular e extravasamento do edema para o interstício, lesando e obstruindo os vasos circundante a retina.

Quando realiza-se o exame de fundoscopia e visualiza o aumento da permeabilidade vascular, considera-se retinopatia leve, quando tem oclusão 
vascular, proliferação ecicatrização, considera-se uma retinopatia moderada a grave. (PEREIRA J. et al.,2020).

Quando o paciente que tem a retinopatia diabética e ainda apresenta a Hipertensão arterial sistêmica (HAS), a lesão nos vasos retiniana lesionaria ainda mais e causaria a isquemiaem alguns casos (PEREIRA J. et al.,2020).

De acordo com a estimativas globais, indicam que 382 milhões de pessoas vivem com DM (8,3\%), e esse número poderá chegar a 592 milhões em 2035, sendo uma importante causade morbidade e mortalidade (GUARIGUATA et al., 2014).

A retinopatia diabética (RD) é considerada para a diabetes mellitus (DM), uma complicação é a principal causa de cegueira, entre 20 e 74 anos de idades. Quando apresenta 20 anos com DM, mais de 90\% dos diabéticos tipo 1 e 60\% tipo 2 apresentarão uma má equidade visual, algum grau de retinopatia diabética (KLEIN et al.,1984; KLEIN et al 2000).

De acordo com a International Diabetes Federation, Agência de Controle da Cegueira, ligada à OMS, e International Federation on Ageing mostra que cinquenta por cento dos diabéticos só é diagnosticada anos depois de conviver com a patologia, pois é uma doença progressiva, acomete aos poucos, podendo ter uma diferença de 29 vezes maior de apresentar perda visual em um paciente com DM do que um paciente não diabético.

Os fatores de risco relacionados a retinopatia diabética (RD), mais relevante é relacionado a duração do diabetes mellitus (DM), quanto mais tempo com a patologia, maior achance de apresentar (BOELTER et al., 2003).

A retinopatia diabética não tem cura, mas pode ser controlada com sessões de fotocoagulação e/ou aplicações de anti - VEGF intravítreo. Em casos mais graves pode ser indicado tratamento cirúrgico (vitrectomia posterior) com objetivo de evitar uma perda visual definitiva (SABROSA, 2013; SILVA et al, 2005).

Portanto, essa doença é de grande relevância, haja vista os prejuízos que a patologia pode causar ao paciente a curto e longo prazo, desde a formação de microaneurismas até a isquemia de determinadas áreas da retina.

Este estudo tem como objetivo coletar informações a respeito da quantidade de pacientes com retinopatia diabética atendidos no Hospital de Olhos do Tocantins da cidade de Araguaína, relacionando sexo, idade, tipo de diabetes, tempo de 
tratamento, diagnóstico de hipertensão e catarata, visto que, essa patologia é a causa mais frequente de cegueira adquirida e é considerada um problema de saúde pública mundial.

\section{METODOLOGIA}

A pesquisa consiste em um estudo de caráter descritivo, transversal e analítico, com análise de prontuários de pacientes atendidos no Hospital de Olhos do Tocantins na cidade de Araguaína -TO no ano de 2020. O acesso ao prontuários foi realizado por meio do Sistema deGestão Hospitalar- Gemmius ${ }^{\circledR}$, utilizando o CID H36.0, específico para patologia em estudo.

Os critérios de inclusão foram os prontuários de pacientes diagnosticados com retinopatia diabética no referido período de estudo e o de exclusão foram os de pacientes diagnosticados com outras patologias, sem associação com a retinopatia diabética.

Os dados foram analisados e digitados em planilha do Microsoft Excel e analisados pelo software Epi Info (Epi Info versão 7.2). Os resultados foram categorizados e apresentados em forma de tabelas.

Avaliou-se a relação entre algumas variáveis estudadas, como a associação entre o tempo de tratamento de diabetes com o tipo de retinopatia, e o uso de insulina com o tipo de retinopatia diabética. O tempo de tratamento foi separado em intervalos de: 0-5 anos, 6-15 anos e mais de 15 anos de tratamento de diabetes. Dentre os tipos de retinopatia diabética tem-se:retinopatia diabética não proliferativa leve, moderada ou severa e retinopatia não proliferativa.Por se tratar de um estudo envolvendo seres humanos, o mesmo foi submetido ao Comitê de ética do Centro Universitário Presidente Antônio Carlos cujo número do parecer de aprovação é 4.436.006 (ANEXO 1). 


\section{RESULTADOS}

$\mathrm{Na}$ Tabela 1 observa-se os resultados relacionados a faixa etária, sexo, diagnóstico de hipertensão arterial e presença de catarata dos 236 pacientes analisados. Observou-se que 115 pacientes (48,73\%) apresentavam idade entre 41 a 60 anos, $112(47,46 \%)$ tinham mais que 60 anos e 9 pacientes $(3,81 \%)$ de 0 a 40 anos. Em relação ao sexo 106 pacientes eram do sexo feminino (44,92\%) e 130 do sexo masculino (55,08\%). Em se tratando de outras patologias associadas, 154 apresentam hipertensão (65,25\%), e 161 (68,22\%) apresentaram diagnóstico de catarata.

Tabela 1: Resultado de sexo, idade, diagnóstico de hipertensão e catarata dos pacientesatendidos no Hospital de Olhos do Tocantins, na cidade de Araguaína no ano de 2020.

\begin{tabular}{|c|c|c|}
\hline Variáveis & $\mathbf{n}$ & $\%$ \\
\hline \multicolumn{3}{|l|}{ Sexo } \\
\hline $\begin{array}{l}\text { Feminino } \\
\text { Masculino }\end{array}$ & $\begin{array}{l}106 \\
130\end{array}$ & $\begin{array}{l}44,92 \\
55,08\end{array}$ \\
\hline \multicolumn{3}{|l|}{ Idade (anos completos) } \\
\hline $\begin{array}{l}0-40 \text { anos } \\
41-60 \text { anos } \\
>60 \text { anos }\end{array}$ & $\begin{array}{c}9 \\
115 \\
112\end{array}$ & $\begin{array}{l}3,81 \% \\
48,73 \% \\
47,46 \%\end{array}$ \\
\hline \multicolumn{3}{|l|}{ Hipertensão } \\
\hline $\begin{array}{l}\text { Sim } \\
\text { Não }\end{array}$ & $\begin{array}{c}154 \\
82\end{array}$ & $\begin{array}{l}65,25 \% \\
34,75 \%\end{array}$ \\
\hline \multicolumn{3}{|l|}{ Catarata } \\
\hline $\begin{array}{l}\text { Sim } \\
\text { Não }\end{array}$ & $\begin{array}{c}161 \\
75\end{array}$ & $\begin{array}{c}68,22 \\
31,78 \%\end{array}$ \\
\hline
\end{tabular}

Na Tabela 2 observa-se a relação do tempo de tratamento de diabetes dos pacientes analisados com o tipo de retinopatia que os mesmos apresentavam. Dentre os pacientes que tratavam a diabetes por até 5 anos, $24(60 \%)$ apresentavam retinopatia diabética não proliferativa leve (RDNP leve), 7 (17,50\%) 
tinham retinopatia diabética não proliferativa moderada (RDNP moderada) e 9 $(22,50 \%)$ tinham retinopatia diabética não proliferativa severa (RDNP severa). Em relação aos que tinham um tempo de tratamento entre 6 e 15 anos, $44(38,94 \%)$ apresentavam retinopatia diabética proliferativa (RDP), $24(21,25 \%)$ retinopatia diabética não proliferativa severa (RDNP severa), 26 (23,01\%) retinopatia diabética nãoproliferativa moderada (RDNP moderada) e 19 (16,81\%) retinopatia diabética não proliferativa leve (RDNP leve). Dentre os que tratavam por mais de 15 anos, 32 $(43,83 \%)$ também estavam com retinopatia do tipo proliferativa (RDP), $15(20,55 \%)$ tinham retinopatia diabética não proliferativa severa (RDNP severa), 15 (20,55\%) com retinopatia diabética não proliferativa moderada (RDNP moderada) e 11 $(15,07 \%)$ com retinopatia diabética não proliferativa leve (RDNP leve). Tabela 2: Relação entre o tempo de tratamento de diabetes e o tipo de retinopatia diabética.

\section{Tipo de Retinopatia diabética}

\begin{tabular}{|c|c|c|c|c|}
\hline Tempo & Leve & Moderada & Severa & Profiferativa \\
\hline \multirow{2}{*}{$0-5$ anos } & 24 & 7 & 9 & 0 \\
\cline { 2 - 5 } & $60 \%$ & $17,50 \%$ & $22,50 \%$ & $0 \%$ \\
\hline \multirow{2}{*}{$6-15$ anos } & 19 & 26 & 24 & 44 \\
\cline { 2 - 5 } & $16,81 \%$ & 23,01 & $21,24 \%$ & $38,94 \%$ \\
\hline \multirow{2}{*}{ >15 anos } & 11 & 15 & 15 & 32 \\
\cline { 2 - 5 } & $15,07 \%$ & $20,55 \%$ & $20,55 \%$ & $43,83 \%$ \\
\hline
\end{tabular}

Na Tabela 3 estão apresentados os resultados da relação do uso de insulina com o tipo de retinopatia diabética. Dos 236 pacientes avaliados, 98 (41,95\%) relataram fazer uso de insulina e destes 48 (48,98\%) apresentavam retinopatia diabética proliferativa (RDP), $21(21,43 \%)$ retinopatia diabética não proliferativa moderada (RDNP moderada), $16(16,33 \%)$ retinopatia diabética não proliferativa severa (RDNP severa) e $13(13,27 \%)$ retinopatia diabética não proliferativa leve (RDNP leve). 
Tabela 3: Relação do uso de insulina com o tipo de retinopatia diabética.

\begin{tabular}{lcc}
\hline Tipo de Retinopatia & $\mathbf{n}$ & $\mathbf{\%}$ \\
\hline RDP & 48 & $48,98 \%$ \\
RDNP Leve & 13 & $13,27 \%$ \\
RDNP Moderada & 21 & $21,43 \%$ \\
RDNP Severa & 16 & $16,33 \%$ \\
\hline Usam insulina & 98 & $100 \%$ \\
\hline
\end{tabular}

\section{DISCUSSÃO}

De acordo com o estudo, na Tabela 1, observa-se uma diferença minuciosa entre os sexos dos pacientes diagnosticados com retinopatia diabética (RD), sendo o sexo masculino mais prevalente com $55,08 \%$. Entretanto, outras pesquisas como a realizada por Garcia et al (2003), a proporção entre homens e mulheres diagnosticados com RD foi de 3:4 respectivamente para o sexo masculino e feminino. Porém Kalter-Leibovici (1997) afirma queo sexo do paciente não aumenta a chance de desenvolver a patologia em estudo.

Segundo Guedes et al (2009) cerca de 1 a $3 \%$ da população mundial apresenta algum grau da retinopatia diabética (RD), sendo a maioria após os 40 de idade e $5 \%$ antes dos 20 anos. Dessa forma, evidencia-se que quanto mais avançada é sua idade, maior a chance de apresentara patologia, o que corrobora a pesquisa apresentada, isso devido ao grande aumento do sedentarismo e alimentação que são consideradas não saudáveis, em excesso por um longo período, além disso aqueles que tem o histórico familiar de diabetes tende a apresentar esta doença e com isso pode ocasionar lesões em vários órgãos, como os rins, nervos periféricos e os olhos.

O estudo de Garcia et al (2003), realizado com 1002 pacientes diagnosticados com retinopatia diabética, mostrou que pacientes abaixo dos 40 anos de idade apresentavam uma menor incidência da doença, e que não houve diferença estatística significativa quanto aos grupos dos pacientes avaliados com idade entre 40 e 60 anos e maior que 60 anos, que eram os mais prevalentes. Esses resultados 
corroboram o presente estudo, uma vez que a maior prevalência da doença ocorreu em pacientes com a faixa etária acima de 40 anos de idade, conforme apresentado na Tabela 1.

Em relação ao diagnóstico de hipertensão arterial sistêmica (HAS) observouse uma forte associação entre hipertensão e retinopatia diabética. De acordo com as Diretrizes Brasileiras de Hipertensão Arterial (HA) publicada no ano de 2020, a hipertensão é caracterizada por elevação persistente da pressão arterial (PA), ou seja, PA sistólica maior ou igual a $140 \mathrm{mmHg}$ e PA diastólica maior ou igual a 90 $\mathrm{mmHg}$, seguindo as técnicas de forma correta, em pelo menos dois momentos diferentes, sem o uso de medicamentos anti- hipertensivo. No olho, essas alterações pressóricas combinadas com o diabetes mellitus são suficientes para causar mudanças na vascularização do segmento posterior da coroide, na retinae na papila óptica, levando ou contribuindo para a patogênese da retinopatia diabética (ISSA, 2020).

A hipertensão causada por diabetes está relacionada com o aumento do conteúdo de $\mathrm{Na}+$ extracelular corporal e expansão de volume e acontece com frequência em DM tipo 1 e DM tipo 2, sendo responsiva a inibição do sistema reninaangiotensina-aldosterona (SRAA).

No DM tipo 1 a hipertensão se manifesta logo após a nefropatia, quando o rim perde a capacidade de excretar água e solutos. Entretanto, no DM tipo 2, a hipertensão acompanha o diagnóstico de DM nos pacientes obesos, idosos e resistentes a insulina. Por exemplo, A tensãomecânica via hipertensão, o excesso de glicose, a inflamação e as EROS aumentam a produçãode angiotensina II em células renais e contribuem para a hiperativação do SRAA.

A ativação anormal do SRAA contribui para vasoconstrição vascular sistêmica e renal, e reabsorção renal de sódio através da interação com o receptor de angiotensina tipo 1 (AT1), via liberação de aldosterona, o que acaba gerando aumento da pressão arterial, pressão intraglomerular e lesão renal. A ativação do SRAA também pode diminuir a sensibilidade a insulina, contribuindo paraa formação de retinopatia diabética no futuro (HAMMER; MCPHEE, 2016).

Sobretudo, a hipertensão arterial sistêmica (HAS) é cerca de duas vezes mais frequente na população com Diabetes Mellitus e tem um papel de destaque na 
fisiopatologia da retinopatiadiabética. Pacientes que portam a diabetes mellitus (DM) tendem apresentar uma hiperperfusão do leito capilar em todos os tecidos. Com o aumento da pressão arterial há também aumento dapressão intraluminar piorando o extravasamento da rede vascular e favorecendo a filtração de proteínas plasmáticas através do endotélio, e sua deposição na membrana basal do capilar, contribuindo ao dano vascular, gerando isquemia e progredindo a Retinopatia diabética (BOELDER et al., 2003).

O diabetes é um dos principais fatores de risco para o desenvolvimento de catarata, sendo o mau controle glicêmico o maior impulsionador de opacidade do cristalino em pacientes diabéticos. Existem poucos estudos na literatura relacionando catarata com DM tipo 1 e tipo 2, porém, estudos epidemiológicos mostram existir alguns fatores que contribuem para o surgimento de catarata como o tempo de duração dessa patologia, a idade avançada, a retinopatia avançada, o uso de diuréticos e o mau controle metabólico (KATO et al, 2001).

A ligação entre DM tipo 2 e catarata torna-se mais difícil de elucidar devido ao passado glicêmico do paciente, a duração da doença e a idade, que combinadas, contribuem para a opacidade do cristalino. Entretanto, no DM tipo 1, a duração da doença e o controle metabólico tende a ser mais determinado. Alguns estudos analisaram o impacto do controle glicêmico em pacientes com DM tipo 1, utilizando densitômetro através do exame de biomicroscopia e determinaram que o principal fator correlacionado com essa patologia seria o valor da HbA1c (hemoglobina glicada), como fator de risco para catarata em pacientes com DM tipo 1 (PIZZOLet al, 2008).

De acordo Silva et al (2005), após 15 anos de diabetes a prevalência de retinopatia entre os portadores de diabetes mellitus insulino-dependentes é de $97 \%$. E sabe-se que a doença apresenta um caráter progressivo, e desta forma se não tratada precocemente pode evoluir paraa forma mais grave, retinopatia proliferativa. Esses dados corroboram com o encontrado no presente estudo, visto que os pacientes com mais de 15 anos de tratamento apresentaram maior prevalência de retinopatia proliferativa.

Outrossim, destaca-se o estudo feito por Nehemy (1998) a respeito do tempo dediagnóstico da diabetes e a prevalência da retinopatia diabética. Estudos 
realizados na década de 80 revela que indivíduos com DM tipo 1, insulino dependente que foi diagnosticado antes dos 30 anos de idade, a retinopatia tem uma variação de $17 \%$ em pacientes com menos de 5 anos de diabetes e $98 \%$ em pacientes com diabetes há mais de 15 anos. Além disso, o estudo mostra que pacientes insulino dependentes, com DM tipo 1, tem uma relação intrínseca com a forma mais grave da retinopatia diabética a RDP. Porém, essas porcentagens sejam mais baixasem pacientes que controlam de forma correta o índice glicêmico.

Vários estudos procuram examinar os fatores de risco para 0 desenvolvimento eprogressão da retinopatia diabética proliferativa. $O$ uso de insulina prolongado parece está ligado com a forma mais grave de retinopatia diabética.

O estudo transversal realizado por Freitas et al. (2002) afirma que existe uma maior prevalência de retinopatia diabética proliferativa em pacientes insulino dependentes comparado aos não insulino dependentes. Todavia, não existe uma explicação elucidada para tal associaçãodevido a diferença metabólica nas DM tipo 1 e 2, ou a disparidade na idade dos pacientes e níveis de glicemia maior na DM tipo 1.

\section{CONCLUSÃO}

Os pacientes que apresentavam mais tempo de tratamento de diabetes mellitus (>15 anos) tinham a maior prevalência em relação à retinopatia do tipo proliferativa que é considerada mais grave. O estudo também confirmou que os pacientes que faziam uso de insulina para o tratamento de diabetes mellitus foram os mais acometidos com a forma mais grave da retinopatia diabética, aumentando desta forma o risco em desenvolver cegueira, caso a patologia não seja tratada.

O presente estudo traz o alerta para a importância do diagnóstico precoce da retinopatia diabética, pois através de um simples exame de fundo de olho, pode-se evitar, com medidas terapêuticas sistêmicas e oculares, a progressão da patologia em questão para as formas mais graves, prevenindo assim uma perda significativa da visão. 


\section{REFERÊNCIAS BIBLIOGRÁFICAS}

BOELTER, M.C; AZEVEDO, M.J; GROSS, J.L; LAVINSKY, J. Fatores de risco para retinopatia diabética. Arquivos Brasileiros de Oftalmologia, v.66, n.2, p.239-247, 2003.

BOSCO, A; LERARIO, A.C: SORIANO, D; SANTOS, R.F; MASSOTE, P; GALVÃO, D; FRANCO, A.C.H.M; PURISCH, S; FERREIRA, A.R. Retinopatia diabética. Arquivos Brasileiros de Endocrinologia \& Metabologia, v.49, n.2, p.217-227, 2005.

FREITAS, André et al. A proteinúria como fator de risco para retinopatia diabética. Arquivos Brasileiros de Oftalmologia.vol.65 no.1 São Paulo Jan./Feb. 2002 https://www.scielo.br/scielo.php?pid=S0004-27492002000100016\&script=sci_arttext GARCIA, CAA; GOMES, AHB; NUNES, IM; OLIVEIRA, TL; MONTEIRO, J. Incidência e fatores de risco da retinopatia diabética em pacientes do Hospital Universitário Onofre Lopes, Natal-RN. Arq. Bras. Oftalmol. V.66, n.3, p.355-358, 2003

GUARIGUATA, L; WHITING, DR; HAMBLETON, I; BEAGLEY, J; LINNENKAMP, U; SHAW, J.E. Global estimates of diabetes prevalence for 2013 and projections for 2035. Diabetes research and clinical practice, v. 103, n. 2, p. 137-149, 2014.

GUEDES, Murielem Fernandes et al. Prevalência da retinopatia diabética em unidade do Programa de Saúde da Família. Rev. bras.oftalmol., Rio de Janeiro, v. 68, n. 2, p. 90-95, Apr. 2009. Available from <http://www.scielo.br/scielo.php?script=sci_arttext\&pid=S003472802009000200005\&lng=en\&nrm=iso $>$. access on 23 Feb. 2021. http://dx.doi.org/10.1590/S0034-72802009000200005.

HAMMER, G. D.; MCPHEE, S. J. Fisiopatologia da doença: uma introdução à medicina clínica. 7. ed. Porto Alegre: AMGH, 2016. p 539.

KALTER-LEIBOVICI O, LEIBOVICI L, LOYA N, KREMER I, AXER-SIEGEL R, KARP M, LARON Z. The development and progression of diabetic retinopathy in type I diabetic patients: a cohort study. Diabet Med, v.14, p.858-866, 1997.

KATO S, SHIOKAWA A, FUKUSHIMA H, NUMAGA J, KITANO S, HORI $\mathrm{S}$, et al. Glycemic control and lens transparency in patients with type 1 diabetes mellitus. Am $\mathbf{J}$ Ophthalmol. v.131(3):301-4, 2001.

KLEIN, R; KLEIN, BE; MOSS, SE. Visual impairment in diabetes. Ophthalmology. 1984;91:1-9.

KLEIN, R; KLEIN, BEK. Epidemiology of Eye Disease in Diabetes. In: Flynn Jr HW, SmiddyWE, editors. Diabetes and Ocular Disease: Past, Present, and Future Therapies. The Foundation of the American Academy of Ophthalmology. v.2, p.19-61, 2000.

ISSA, A. F. C., NASCIMENTO, B. R., CORREA FILHO, H., VIEIRA, M. L. C., BRROSO, W. K. S., RODRIGUES, C. S; MOTA-GOMES, M. A. Diretrizes Brasileiras de HipertensãoArterial2020.

MCLELLAN, K.C.P; BARBALHO, S.M; CATTALINI, M; LERARIO, A.C. Diabetes mellitus do tipo 2, síndrome metabólica e modificação no estilo de vida. Campinas, v.20, n.5. Sept./Oct. 2007.

PEREIRA, J.A; BERTOLI, M.A.T; PEREIRA, G.D.C; CORGOZINHO, L.C. Atualizações sobre retinopatia diabética: uma revisão narrativa. Revista Eletrônica Acervo Saúde, n.49, p.3428- 
3428, 2020.

PIZZOL, M.M.D; ESTEVES, J.F; SCCOCO, C.A; ROGGIA, M.F; ROSA, C.M; LAMBERT, J.H.F; CANINI, L.H. Catarata e diabetes mellitus tipo 1. Arquivos Brasileiros de Oftalmologia, 71(4), 564-567, 2008.

SBD - Sociedade Brasileira de Diabetes. Diretrizes da Sociedade Brasileira de Diabetes: 2014-2015. São Paulo: Clannad. SEABRA, A.L.R, 2015.

SILVA, Valdir Balarin et al. Tratamento da retinopatia diabética: percepções de pacientes emRio Claro (SP) - Brasil. Arq. Bras. Oftalmol., São Paulo, v. 68, n. 3, p. 363-368, June 2005 .Available from <http://www.scielo.br/scielo.php?script=sci_arttext\&pid=S000427492005000300016\&Ing=en\&nrm=iso $>$. access on $23-$ Feb. 2021. https://doi.org/10.1590/S0004-27492005000300016. 


\section{ANEXOS \\ CENTRO UNIVERSITÁRIO TOCANTINENSE PRESIDENTE ANTÔNIO CARLOS - UNITPAC PARECER CONSUBSTANCIADO DO CEP}

\section{DADOS DO PROJETO DE PESQUISA}

Título da Pesquisa: Avaliação epidemiológica de pacientes com retinopatia diabética no hospital de olhos doTocantins da cidade de Araguaína-TO no período de 2019 a 2020.

Pesquisador: Rejanne Lima Arruda

Área Temática:

Versão: 3

CAAE: 37284620.3 .0000 .0014

Instituição Proponente: FUNDO MUNICIPAL DE SAUDE

Patrocinador Principal: Financiamento Próprio

\section{DADOS DO PARECER}

Número do Parecer: 4.436.006

\section{Apresentação do Projeto:}

O projeto objetiva-se em analisar os dados epidemiológicos dos pacientes atendidos no Hospital de Olhos doTocantins no período de Janeiro 2019 a Dezembro 2020.

\section{Objetivo da Pesquisa:}

Analisar os dados epidemiológicos dos pacientes com retinopatia diabética, no Hospital de olhos doTocantins, na cidade de Araguaína-TO no período de 2019 a 2020.

\section{Avaliação dos Riscos e Benefícios:}

A avaliação dos riscos e benefícios está conforme os termos dispostos na Res. CNS $466 / 2012$

\section{Comentários e Considerações sobre a Pesquisa:}

Altamente relevante para a comunidade cientifica. A pesquisa tem sua relevância ao propor um estudo da retinopatia diabética em pacientes atendidos no Hospital de olhos do Tocantins servirá de base para o levantamento e avaliação dos principais grupos de risco, assim como verificação das medidas preventivas para a redução do número de casos no Estado, e contribuição com o aumento de publicações nessa área. 
Considerações sobre os Termos de apresentação obrigatória: Os termos foram apresentados conforme as Resolução CNS e suas atualizaçoes.

\section{Recomendações:}

Segundo Carta Circular n. 039/2011/CONEP/CNS/GB/MS, de 30 de setembro de 2011, contudonão cabe ao Sistema CEP/CONEP legislar sobre o acesso e USO DO PRONTUÁRIO médico (entenda-se odontológico também), porém cabe determinar o cumprimento do sigilo e da confidencialidade, além de exigir que toda pesquisa envolvendo seres humanos trate os mesmos em sua dignidade, respeite-os em sua autonomia e defenda-os em sua vulnerabilidade, conformeResolução CNS 466/12

\section{Conclusões ou Pendências e Lista de Inadequações:}

Recomendo aprovação do projeto.

\section{Considerações Finais a critério do CEP:}

O Colegiado vota com o relator.

Este parecer foi elaborado baseado nos documentos abaixo relacionados:

\begin{tabular}{|c|c|c|c|c|}
\hline $\begin{array}{c}\text { Tipo } \\
\text { Documento }\end{array}$ & Arquivo & Postagem & Autor & Situação \\
\hline \begin{tabular}{|l|} 
Informações \\
Básicas do \\
Projeto \\
\end{tabular} & $\begin{array}{l}\text { PB_INFORMAÇÕES_BÁSICAS_DO } \\
\text { PROJETO_1624122.pdf }\end{array}$ & $\begin{array}{l}16 / 11 / 2020 \\
14: 59: 24\end{array}$ & & Aceito \\
\hline $\begin{array}{|lr|}\text { TCLE } & \\
\text { Termos r de } \\
\text { Assentiment } \\
0 \\
\text { Justificativa } \\
\text { de Ausência } \\
\end{array}$ & TCLERETINOPATIA1310.pdf & $\begin{array}{c}15 / 10 / 2020 \\
07: 53: 49\end{array}$ & $\begin{array}{l}\text { Rejanne } \\
\text { Lima } \\
\text { Arruda }\end{array}$ & Aceito \\
\hline Outros & $\begin{array}{l}\text { declaracaodeusodedadosemateriais. } \\
\text { pdf }\end{array}$ & $\begin{array}{c}02 / 09 / 2020 \\
10: 59: 07\end{array}$ & $\begin{array}{l}\text { Rejanne } \\
\text { Lima } \\
\text { Arruda }\end{array}$ & Aceito \\
\hline Outros & declaracaodepublicidade.pdf & $\begin{array}{c}02 / 09 / 2020 \\
10: 58: 19 \\
\end{array}$ & $\begin{array}{l}\text { Rejanne } \\
\text { Lima } \\
\text { Arruda } \\
\end{array}$ & Aceito \\
\hline Outros & cartadeencaminhamento.pdf & $\begin{array}{c}02 / 09 / 2020 \\
10: 58: 02 \\
\end{array}$ & \begin{tabular}{|l} 
Rejanne \\
Lima \\
Arruda \\
\end{tabular} & Aceito \\
\hline \begin{tabular}{|l|} 
Projeto \\
Detalhado \\
Brochur a \\
Investig ador \\
\end{tabular} & TCCRETINOPATIA.pdf & $\begin{array}{l}\text { 02/09/2020 } \\
10: 43: 08\end{array}$ & $\begin{array}{l}\text { Rejanne } \\
\text { Lima } \\
\text { Arruda }\end{array}$ & Aceito \\
\hline Outros & 20200831153610825_0001.pdf & $\begin{array}{c}02 / 09 / 2020 \\
10: 42: 13\end{array}$ & $\begin{array}{l}\text { Rejanne } \\
\text { Lima } \\
\text { Arruda }\end{array}$ & Aceito \\
\hline
\end{tabular}




\begin{tabular}{|l|l|l|l|l|}
\hline $\begin{array}{l}\text { Folha } \\
\text { Rosto }\end{array}$ & Folhaderosto.pdf & $\begin{array}{c}02 / 09 / 2020 \\
10: 41: 47\end{array}$ & $\begin{array}{l}\text { Rejanne } \\
\text { Lima } \\
\text { Arruda }\end{array}$ & Aceito \\
\hline
\end{tabular}

Situação do Parecer:

Aprovado

Necessita Apreciação da CONEP:

Não

Assinado por:
MARGARIDA DO SOCORROSILVA ARAUJO
(Coordenadora)

